\title{
Wireless Energy Harvesting Using Signals from Multiple Fading Channels
}

\author{
Yunfei Chen, Senior Member, IEEE, Nan Zhao, Senior Member, IEEE, and Mohamed-Slim Alouini, Fellow, IEEE
}

\begin{abstract}
In this paper, we study the average, the probability density function and the cumulative distribution function of the harvested power. In the study, the signals are transmitted from multiple sources. The channels are assumed to be either Rician fading or Gamma-shadowed Rician fading. The received signals are then harvested by using either a single harvester for simultaneous transmissions or multiple harvesters for transmissions at different frequencies, antennas or time slots. Both linear and nonlinear models for the energy harvester at the receiver are examined. Numerical results are presented to show that, when a large amount of harvested power is required, a single harvester or the linear range of a practical nonlinear harvester are more efficient, to avoid power outage. Further, the power transfer strategy can be optimized for fixed total power. Specifically, for Rayleigh fading, the optimal strategy is to put the total power at the source with the best channel condition and switch off all other sources, while for general Rician fading, the optimum magnitudes and phases of the transmitting waveforms depend on the channel parameters.
\end{abstract}

Index Terms-Cumulative distribution function, energy harvesting, fading channels, nonlinear distortion, probability density function, shadowing.

\section{INTRODUCTION}

As a promising solution to the energy shortage problem in portable devices, wireless energy harvesting allows for sustained operation. In wireless energy harvesting, the portable device is equipped with an energy harvester and thus, it can collect energy from either dedicated or ambient sources to replenish its energy supply. For example, in [1], energy harvesting from ambient radio frequency signals in several commonly used frequency bands was investigated. The amount of power available from such ambient sources is often at the scale of milli-watts or micro-watts [2]. Also, in [3], signals transmitted by peer nodes in the same network were used as ambient sources for energy harvesting at the idle nodes. The ambient energy harvesting depends heavily on the radio environment where the harvester operates and when it operates. Although one may perform scheduling or take other

Manuscript received May 13, 2017; revised June 7, 2017; accepted July 25, 2017. This research was supported in part by the Xinghai Scholars Program, in part by the Fundamental Research Funds for the Central Universities under DUT17JC43, and in part by the National Natural Science Foundation of China (NSFC) under Grant 61671101. The associate editor coordinating the review of this paper and approving it for publication was Z. Zhang. (Corresponding author: Nan Zhao.)

Yunfei Chen is with the School of Engineering, University of Warwick, Coventry, U.K. CV4 7AL (e-mail: Yunfei.Chen@warwick.ac.uk)

Nan Zhao is with the School of Inform. and Commun. Eng., Dalian University of Technology, Dalian, 116024, P. R. China (e-mail:zhaonan@dlut.edu.cn)

Mohamed-Slim Alouini is with the EE program, King Abdullah University of Science and Technology, Thuwal, Mekkah Province, Saudi Arabia (e-mail: slim.alouini@kaust.edu.sa). measures for best performance [4] - [6], there is still great uncertainty in the amount of ambient energy. For applications that require regular power supply, such as mobile services, this uncertainty is not desirable. In these applications, dedicated sources can be used. For example, in [7], a power beacon was proposed that constantly broadcasts wireless power in a cellular network for harvesting. These power beacons are deployed in conjunction with base stations to provide power coverage and signal coverage in the network. In [8], a hybrid access point was proposed where the access point broadcasts wireless power in the downlink followed by data transmission using the harvested energy in the upper link in a time-division duplex manner. In [9], simultaneous wireless information and power transfer was proposed, where the wireless signal is either switched in the time domain or split in the power domain to provide signal transmission and power transfer using the same wireless carrier.

All these works have provided very useful solutions to the energy supply problem of portable devices. However, an important issue that has been largely ignored in these works is the amount of harvested energy available to the portable device for operation and its modeling. Firstly, most wireless signals suffer from fading and/or shadowing during the transmission [10]. This makes the received signal random. This randomness will affect the amount of the harvested energy. Such randomness was investigated in [11] in terms of battery recharging time, but with limited discussion. Secondly, the received energy will only become usable when it is converted by an energy harvester. Hence, the harvester characteristics will determine the amount of the harvested energy. Most existing works assumed a linear input-output relationship for the energy harvester [4] - [9]. However, practical harvesters have nonlinear relationships. For example, based on two practical harvesters in [12] and [13], reference [14] proposed a logarithmic inputoutput relationship. Also, using [12] and other references [15] - [18], a rational input-output relationship was proposed in [19]. In both the logarithmic and rational relationships, the output saturates when the input increases, while in the linear relationship, the output does not. Finally, a single source with a single energy harvester may not be enough for energy supply. Therefore, multiple sources and/or multiple harvesters can be used. For example, two rectifiers operating at different frequencies can be used [18]. Multiple antennas can also be used to harvest more energy from either multiple sources or a single source [20]. As well, one can use one energy harvester to harvest energies from multiple sources, such as co-channel interference [21], [22]. The number of energy sources and the number of energy harvesters will determine the amount of the 
harvested energy. None of these issues have been thoroughly investigated in the literature.

In this paper, we will fill this gap in the literature by providing a comprehensive framework on the analysis and the modeling of the amount of harvested power at the portable device. In the analysis, we consider the case when the wireless signal suffers from Rician fading as well as the case when the wireless signal suffers from both Rician fading and Gamma shadowing. The first case occurs in applications when power transfer is performed in a short distance such that shadowing can be ignored, while the second case occurs in applications when power transfer is performed in longer distances such that the shadowing effect needs to be included. Both linear energy harvester (LEH) and nonlinear energy harvester (NLEH) are studied. The average, the probability density function (PDF) and the cumulative distribution function (CDF) of the harvested power are derived. Based on this derivation, the power outage probability is calculated. Also, optimal power transfer strategies that maximize the average harvested power are discussed. Numerical results are presented to show that different power transfer strategies should be used for different channel conditions and different harvesters in order to maximize the average harvested power. Our results also show that the power outage probability depends on the number of sources, the channel condition and the harvester characteristics, giving us guidelines on how to choose these parameters to meet the outage requirement. The main contributions of this work can be summarized as follows:

- We study the effect of Rician fading and/or Gamma shadowing on the amount of harvested power. In the study, we provide a comprehensive analytical framework by deriving the average, the PDF and the CDF of the harvested power for the case when multiple sources or multiple harvesters are used. These results have not been obtained in the literature.

- We examine the effect of the harvester characteristics on the amount of the harvested power. To the best of the authors' knowledge, this is the first time that the inputoutput relationship of practical energy harvesters is taken into account in the modeling of harvested power.

- We provide useful guidance on the design of wireless energy harvesting systems by examining the power outage requirement for communications and the optimal power transfer strategies for energy harvesting.

The rest of the paper is organized as follows. In Section II, the system model used in the analysis is introduced. Section III studies the case when the wireless signal suffers from fading. Section IV studies the case when the wireless signal suffers from both fading and shadowing. In Section V, applications of the results obtained in Sections III and IV are discussed using the probability of power outage and the power transfer strategy. Numerical examples are presented in Section VI. Finally, some concluding remarks are made in Section VII.

\section{SySTEM MOdEL}

Consider a wireless energy harvesting system where there are $I$ energy sources and $I$ is the total number of sources with $I>1$. Each source transmits an energy signal with a complex amplitude of $x_{i}=\omega_{i} e^{j \psi_{i}}$, where $i=1,2, \cdots, I$ is the source index, $j=\sqrt{-1}, \omega_{i}$ is the magnitude and $\psi_{i}$ is the phase of the transmitted waveform, respectively. Note that for energy transfer $\omega_{i}$ and $\psi_{i}$ could be any values, while for information transmission with modulation they are discrete values determined by the constellation. These signals suffer from either Rician fading or Gamma-shadowed Rician fading in the channels. The faded/shadowed signals are received and then harvested by either a single harvester for simultaneous transmissions or multiple harvesters for transmissions over different frequencies, antennas or time slots.

\section{A. Harvested Power}

If all the sources transmit signals simultaneously in the same frequency band, only a single harvester is required to harvest the energy. The received signal at this harvester can be expressed as

$$
y=\sum_{i=1}^{I} x_{i} h_{i}+n
$$

where $h_{i}$ is the fading coefficient from the $i$-th source to the energy harvester and $n$ is the additive white Gaussian noise (AWGN). The channel gains $h_{i}$ in the simultaneous transmissions are different because the signals are from different sources. In our paper, we assume Rician fading such that $h_{i}$ is a circularly symmetric complex Gaussian random variable with mean $s_{i}=\epsilon_{i} e^{j \phi_{i}}$ and variance $2 \alpha_{i}^{2}$ and its real and imaginary parts are independent of each other, $\epsilon_{i}$ is the magnitude of the mean, $\phi_{i}$ is the phase of the mean, and $n$ is a complex Gaussian random variable with mean zero and variance $2 \beta^{2}$ as well as independent real and imaginary parts. This is the case when the harvester harvests energy from simultaneous transmissions, such as two-way relaying or nonorthogonal multiple access, or from co-channel interference [21], [22]. This is also the case when the power beacons proposed in [7] are deployed in several locations to provide power coverage for the whole network such that one portable device may be served by several power beacons or when distributed antenna systems are adopted, similar to [23] but for power transfer.

Using (1), the received power or the input power of the energy harvester is given by

$$
P_{n}=|y|^{2}=\left|\sum_{i=1}^{I} x_{i} h_{i}+n\right|^{2}
$$

and the harvested power is given by

$$
P_{o}=f\left(P_{n}\right)
$$

where $f(x)$ is the input-output relationship of the energy harvester to be discussed later. In this case, only one energy harvester is required to collect energies from multiple sources.

On the other hand, if these sources operate in different frequency bands, on different antennas or during different time slots, $I$ energy harvesters, or one harvester for $I$ times, are 
required to collect all these energies. The received signal at the $i$-th energy harvester can be given by

$$
y_{i}=x_{i} h_{i}+n_{i}
$$

where $n_{i}$ is the AWGN at the $i$-th energy harvester as a complex Gaussian random variable with mean zero and variance $2 \beta^{2}$, and all the other symbols are defined as before. This is, for example, the case when rectennas tuned at different frequencies [18] or multiple antennas [20] are used to increase the amount of harvested energy, or when the energy is accumulated from transmissions in different time slots.

Using (4), the received or input power of the $i$-th energy harvester can be obtained as

$$
P_{i}=\left|y_{i}\right|^{2}=\left|x_{i} h_{i}+n_{i}\right|^{2}
$$

and the total harvested power after combining all output power of the $I$ energy harvesters is

$$
P_{o}=\sum_{i=1}^{I} f_{i}\left(P_{i}\right)
$$

where $f_{i}(x)$ is the input-output relationship of the $i$-th energy harvester. Note that, in practice, to transfer a fixed amount of energy, we can choose to send it at the full power and collect it using a single energy harvester, or we can choose to split it into several smaller signals and collect them using multiple harvesters. The purpose of this paper is to derive the statistical models of the output power $P_{o}$ in different cases to provide theories for these design issues. Before doing this, we need to discuss the input-output relationship of the energy harvester.

\section{B. Energy Harvester Models}

In most existing works on energy harvesting, the LEH model has been used. In this case, one has the input-output relationship of the energy harvester as [4] - [9]

$$
f(x)=\eta x
$$

where $\eta$ is the conversion efficiency of the energy harvester and $x$ is the input power.

An important assumption here is that the conversion efficiency is a constant that is independent of the input power. However, many measurement data have revealed that the conversion efficiency actually depends on the input power. Consequently, the relationship between the input power and the output power is nonlinear. For example, in reference [19], after examining dozens of practical energy harvesters, the conversion efficiency of the energy harvester was given as a function of the input power $\eta(x)=\frac{p_{2} x^{2}+p_{1} x+p_{0}}{q_{3} x^{3}+q_{2} x^{2}+q_{1} x+q_{0}}$, where $p_{0}, p_{1}, p_{2}, q_{0}, q_{1}, q_{2}, q_{3}$ are constants determined by curvefitting in [19]. Thus, for these energy harvesters [12], [15] - [18], the input-output relationship will be [19]

$$
f(x)=\eta(x) x=\frac{p_{2} x^{3}+p_{1} x^{2}+p_{0} x}{q_{3} x^{3}+q_{2} x^{2}+q_{1} x+q_{0}} .
$$

From (8), unlike the linear model where the output power increases linearly with the input power, the output power of the nonlinear model actually approaches an upper limit of $p_{2} / q_{3}$ when the input power increases, due to the nonlinear distortion
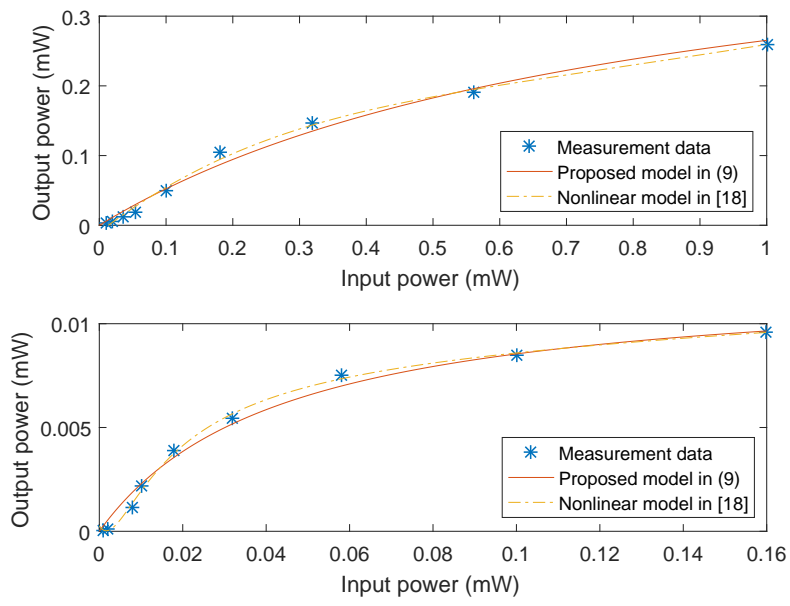

Figure 1. Comparison of measurement, the proposed model (9) and the existing model [19].

or saturation. In another independent work [14], using two measurement data in [12] and [13], the authors also proposed a nonlinear model as $f(x)=\frac{\frac{M}{1+e^{-a(x-b)}}-M \Omega}{1-\Omega}$, where $\Omega=\frac{1}{1+e^{a b}}$ and $a, b$ and $M$ are constants determined by curve-fitting in [14]. This model also captures the nonlinear characteristics of the energy harvester. However, these two models are not mathematically tractable for the derivations of the average, the PDF and the CDF of $P_{o}$ that are of interest in this paper. Thus, we propose a simpler nonlinear model as a modification to the model in (8) by

$$
f(x)=\frac{a x+b}{x+c}-\frac{b}{c}
$$

where $a, b, c$ are constants determined by standard curvefitting, similar to [19] and [14]. Compared with the linear model in (7), the proposed model in (9) can describe the nonlinear distortion or saturation, similar to those in [14] and [19]. Compared with the nonlinear models in [14] and [19], the proposed model in (9) is more mathematically tractable for the derivation of the average, PDF and CDF of the harvested power, as will be shown later. Thus, it is simpler. Also, (7) can be derived from (8) by removing the higher order terms in the numerator and denominator, when $x$ is very small. This is the case in energy harvesting, as the input power is usually quite small. Also, the term of $\frac{b}{c}$ is added to make the output power zero when the input power is zero.

Fig. 1 compares the measurement data with the proposed model in (9) and the nonlinear model in [19]. The upper part of the figure uses the data from [12, Fig. 19], while the lower part of the figure uses data from [15, Fig. 5]. The fitted parameters of the proposed model are $a=2.463, b=1.635, c=0.826$ for [12] with a root mean squared error (RMSE) of 0.009737 and $a=0.3929, b=0.01675$ and $c=0.04401$ for [15] with a RMSE of 0.0003993 . The fitted parameters of the nonlinear model in [19] are $p_{2}=0.1328, p_{1}=0.08107, p_{0}=0.3493$, $q_{3}=0.03764, q_{2}=0.8472, q_{1}=0.7269$ and $q_{0}=0.5604$ for [12] with a RMSE of 0.01731 and $p_{2}=1.137, p_{1}=-0.5553$, $p_{0}=0.1115, q_{3}=0.4175, q_{2}=0.8971, q_{1}=2.079$ and $q_{0}=0.5249$ for [12] with a RMSE of 0.000788 . The fitting 
errors of the proposed (9) and the nonlinear model in [19] are comparable. For example, [19] has a RMSE of 0.000788 , while (9) has a RMSE of 0.0003993 , for [15]. In the following, we will use (9) and denote it as the NLEH model. Although the LEH model is an ideal model, we will still consider it in the following for two reasons. Firstly, the LEH model is a simple model that can provide a good approximation to the linear range of a practical nonlinear harvester. Secondly, most existing works on wireless energy harvesting have assumed the LEH model. By deriving results for the LEH model, it is easier for these existing works to gain insights.

\section{FADING SIGNALS}

In this section, we derive the average, the PDF and the CDF of the harvested power for the case when the wireless signals suffer from Rician fading only.

\section{A. Single Harvester}

In this case, multiple sources are harvested by a single energy harvester. Thus, the output power for the LEH model becomes

$$
P_{S L 1}=\eta P_{n}
$$

and the output power for the NLEH model becomes

$$
P_{S N 1}=\frac{a P_{n}+b}{P_{n}+c}-\frac{b}{c} .
$$

From (1), the received signal $y$ is a complex Gaussian random variable with

$$
\begin{aligned}
& \mu=E\{y\}=\sum_{i=1}^{I} x_{i} s_{i} \\
& 2 \sigma^{2}=E\left\{|y-\mu|^{2}\right\}=2 \sum_{i=1}^{I}\left|x_{i}\right|^{2} \alpha_{i}^{2}+2 \beta^{2} .
\end{aligned}
$$

Thus, $P_{n}=|y|^{2}$ is a non-central chi-square random variable [24]. Using (10) and the PDF and CDF of $P_{n}$, the PDF and CDF of the output power for the LEH model can be derived via the variable transformation $y=\eta x$ as

$$
\begin{aligned}
& f_{P_{S L 1}}(y)=\frac{1}{2 \eta \sigma^{2}} e^{-\frac{\eta|\mu|^{2}+y}{2 \eta \sigma^{2}}} I_{0}\left(\frac{\sqrt{y}|\mu|}{\sqrt{\eta} \sigma^{2}}\right) \\
& F_{P_{S L 1}}(y)=1-Q_{1}\left(\frac{|\mu|}{\sigma}, \frac{\sqrt{y}}{\sqrt{\eta} \sigma}\right)
\end{aligned}
$$

respectively, where $I_{0}(\cdot)$ is the zero-th order modified Bessel function of the first type [25] and $Q_{1}(\cdot, \cdot)$ is the first-order Marcum Q function [10].

Also, using (11), the PDF and CDF of the output power for the NLEH model can be derived using the variable transformation $y=\frac{a x+b}{x+c}-\frac{b}{c}$ as

$$
\begin{aligned}
f_{P_{S N 1}}(y)= & \frac{1}{2 \sigma^{2}} e^{-\frac{|\mu|^{2}+c y /\left(a-\frac{b}{c}-y\right)}{2 \sigma^{2}}} I_{0}\left(\sqrt{\frac{c y}{a-\frac{b}{c}-y}} \frac{|\mu|}{\sigma^{2}}\right) \\
& \frac{a c-b}{\left(a-\frac{b}{c}-y\right)^{2}} \\
F_{P_{S N 1}}(y)= & 1-Q_{1}\left(\frac{|\mu|}{\sigma}, \frac{\sqrt{c y}}{\sqrt{\left(a-\frac{b}{c}-y\right)} \sigma}\right)
\end{aligned}
$$

respectively.

Finally, the average output power for the LEH model can be calculated as

$$
E\left\{P_{S L 1}\right\}=\eta\left[\left|\sum_{i=1}^{I} x_{i} s_{i}\right|^{2}+2 \sum_{i=1}^{I}\left|x_{i}\right|^{2} \alpha_{i}^{2}+2 \beta^{2}\right]
$$

by using the moments of a non-central chi-square random variable $|y|^{2}$ [24]. For the NLEH model, the average output power can be calculated as

$$
E\left\{P_{S N 1}\right\}=\frac{1}{2 \sigma^{2}} \int_{0}^{\infty}\left(\frac{a x+b}{x+c}-\frac{b}{c}\right) e^{-\frac{|\mu|^{2}+x}{2 \sigma^{2}}} I_{0}\left(\frac{\sqrt{x}|\mu|}{\sigma^{2}}\right) d x
$$

where we have used the PDF of $P_{n}$. To obtain a closed-form expression, first, the approximation to the Bessel functions proposed in [26] is used as

$$
I_{0}(x) \approx \sum_{l=1}^{n_{0}} \frac{\Gamma\left(n_{0}+l\right) n_{0}^{1-2 l}}{\Gamma(l+1)^{2} \Gamma\left(n_{0}-l+1\right)}\left(\frac{x}{2}\right)^{2 l}
$$

where $\Gamma(\cdot)$ is the Gamma function [25] and $2 n_{0}$ is the order of a Chebyshev polynomial used to approximate the Bessel function [26]. It was reported in [26] that this approximation is very accurate for small values of $x$, which is the case for the wireless signal received by the energy harvester. For example, for $I_{0}(x)$, the error is less than $4 \%$ when $0<x<12$ and $n_{0}=20$ [26, Fig. 2]. We use $n_{0}=20$ in the following. Then, using (19) in (18) and solving the integral using [27, eq. (3.383.10)], one has

$$
\begin{aligned}
E\left\{P_{S N 1}\right\} & \approx a-\frac{b}{c}+\frac{b-a c}{2 \sigma^{2}} e^{-\frac{|\mu|^{2}}{2 \sigma^{2}}+\frac{c}{2 \sigma^{2}}} \\
\sum_{l=0}^{n_{0}} & \frac{\Gamma\left(n_{0}+l\right) n_{0}^{1-2 l}}{\Gamma(l+1) \Gamma\left(n_{0}-l+1\right)}\left(\frac{c|\mu|^{2}}{4 \sigma^{4}}\right)^{l} \Gamma\left(-l, \frac{c}{2 \sigma^{2}}\right)
\end{aligned}
$$

for the NLEH model, where $\Gamma(\cdot, \cdot)$ is the upper incomplete Gamma function [25]. Next, we study the case when multiple harvesters are used.

\section{B. Multiple Harvesters}

In this case, $I$ energy harvesters, or one energy harvester for $I$ times, are used to collect energies. If the LEH model is used, the harvested power is given by

$$
P_{M L 1}=\sum_{i=1}^{I} \eta_{i} P_{i}
$$

where $\eta_{i}$ is the conversion efficiency of the $i$-th energy harvester, and if the NLEH model is used,

$$
P_{M N 1}=\sum_{i=1}^{I}\left[\frac{a_{i} P_{i}+b_{i}}{P_{i}+c_{i}}-\frac{b_{i}}{c_{i}}\right]
$$

where $a_{i}, b_{i}$ and $c_{i}$ are the constants for the $i$-th energy harvester.

From (4), $y_{i}$ is a complex Gaussian random variable with

$$
\begin{aligned}
& \mu_{i}=E\left\{y_{i}\right\}=x_{i} s_{i} \\
& 2 \sigma_{i}^{2}=E\left\{\left|y_{i}-\mu_{i}\right|^{2}\right\}=2\left|x_{i}\right|^{2} \alpha_{i}^{2}+2 \beta^{2}
\end{aligned}
$$

Thus, $P_{i}=\left|y_{i}\right|^{2}$ in (21) and (22) is a non-central chi-square random variable. 
Using the linearity of expectation and the moments of the non-central chi-square random variables $P_{i}$, the average harvested power for the LEH model can be derived as

$$
E\left\{P_{M L 1}\right\}=\sum_{i=1}^{I}\left[\eta_{i}\left|x_{i}\right|^{2}\left|s_{i}\right|^{2}+2 \eta_{i}\left|x_{i}\right|^{2} \alpha_{i}^{2}+2 \beta^{2} \eta_{i}\right] .
$$

Similarly, the average harvested power for the NLEH model can be derived by using (19) and [27, eq. (3.383.10)] as

$$
\begin{aligned}
E\left\{P_{M N 1}\right\} \approx & \sum_{i=1}^{I}\left(a_{i}-\frac{b_{i}}{c_{i}}\right)+\sum_{l=0}^{n_{0}} \sum_{i=1}^{I} \frac{\Gamma\left(n_{0}+l\right) n_{0}^{1-2 l}\left(\frac{c_{i}\left|\mu_{i}\right|^{2}}{4 \sigma_{i}^{4}}\right)^{l}}{\Gamma(l+1) \Gamma\left(n_{0}-l+1\right)} \\
& \frac{b_{i}-a_{i} c_{i}}{2 \sigma_{i}^{2}} e^{-\frac{\left|\mu_{i}\right|^{2}}{2 \sigma_{i}^{2}}+\frac{c_{i}}{2 \sigma_{i}^{2}}} \Gamma\left(-l, \frac{c_{i}}{2 \sigma_{i}^{2}}\right)
\end{aligned}
$$

where all the symbols are defined as before. Next, we derive the PDF and CDF.

For (21), the output power can be rewritten as

$$
P_{M L 1}=\sum_{i=1}^{I} \eta_{i} \sigma_{i}^{2} t_{i}
$$

where $t_{i}$ is a non-central chi-square random variable with $v_{i}=2$ degrees of freedom and non-centrality parameter $\delta_{i}=\frac{\left|x_{i}\right|^{2}\left|s_{i}\right|^{2}}{\sigma_{i}^{2}}$. Thus, $P_{M L 1}$ is a sum of weighted non-central chi-square random variables. The exact PDF of $P_{M L 1}$ has been derived in [28]. However, its form is not mathematically convenient, as it requires the calculation of a series whose converging rate needs to be controlled by choosing two parameters heuristically, in addition to the complicated coefficients of the series that need to be determined. Thus, we look for mathematically convenient approximations to the distribution of $P_{M L 1}$. One such approximation was proposed in [29], where a non-central chi-square distribution was used to approximate the sum $P_{M L 1}$. This approximation is still relatively complicated, as the $\mathrm{CDF}$ of a non-central chi-square random variable does not have a closed-form expression except for some special cases. A simpler alternative is momentmatching. To use this method, the mean and variance of $P_{M L 1}$ can be derived from (21) using moments of a non-central chisquare random variable as

$$
\begin{array}{r}
m_{1}=E\left\{P_{M L 1}\right\}=\sum_{i=1}^{I} \eta_{i} \sigma_{i}^{2}\left[v_{i}+\delta_{i}\right] \\
m_{2}=E\left\{\left(P_{M L 1}-m_{1}\right)^{2}\right\}=\sum_{i=1}^{I}\left(\eta_{i} \sigma_{i}^{2}\right)^{2}\left[2 v_{i}+4 \delta_{i}\right]
\end{array}
$$

where $v_{i}$ and $\delta_{i}$ are the degree of freedom and the noncentrality parameter of $t_{i}$, respectively, as given before. By matching them to the mean and variance of a Gamma distribution, one has the Gamma approximation as

$$
\begin{aligned}
& f_{P_{M L 1}}(y) \approx \frac{1}{\theta_{M L 1}^{k_{M L 1}} \Gamma\left(k_{M L 1}\right)} y^{k_{M L 1}-1} e^{-\frac{y}{\theta_{M L 1}}} \\
& F_{P_{M L 1}}(y) \approx \frac{1}{\Gamma\left(k_{M L 1}\right)} \gamma\left(k_{M L 1}, \frac{y}{\theta_{M L 1}}\right)
\end{aligned}
$$

where $k_{M L 1}=\frac{m_{1}^{2}}{m_{2}}$ and $\theta_{M L 1}=\frac{m_{2}}{m_{1}}$ from $k_{M L 1} \theta_{M L 1}=m_{1}$ and $k_{M L 1} \theta_{M L 1}^{2} \stackrel{m_{2}}{=} m_{2}$, and $\gamma(\cdot, \cdot)$ is the lower incomplete

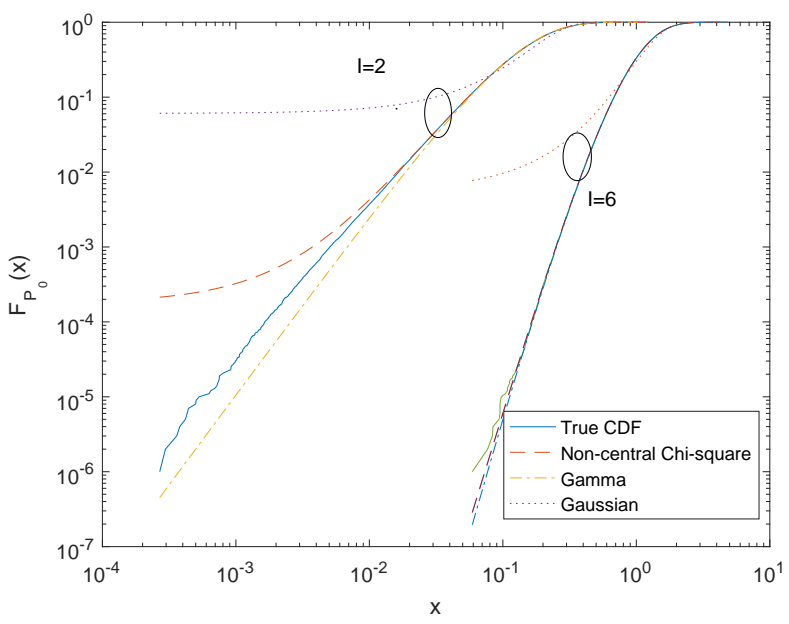

Figure 2. Comparison of different approximations to the CDF of $P_{M L 1}$ in (26).

Gamma function [25]. Also, one can match them to the mean and variance of a Gaussian distribution for the Gaussian approximation.

Fig. 2 compares different approximations to the CDF of $P_{M L 1}$ in (26). In this figure, we set $x_{i}=1,2 \alpha_{i}^{2}=s_{i}=$ $0.1 i \mathrm{~mW}, \beta^{2}=-40 \mathrm{dBm}$, and $\eta=0.3$. One sees that the Gamma distribution provides the highest overall accuracy. Since it also has a simpler CDF, in the following, we will use the Gamma approximation in (28) and (29) for $P_{M L 1}$.

For the output power of the NLEH model in (22), we also use moment-matching approximations. In this case, one can obtain the mean and variance from (22) as

$$
\begin{aligned}
& r_{1}=E\left\{P_{M N 1}\right\}=\sum_{i=1}^{I} r_{1 i} \\
&=\sum_{i=1}^{I}\left[\left(a_{i}-\frac{b_{i}}{c_{i}}\right)+\frac{b_{i}-a_{i} c_{i}}{2 \sigma_{i}^{2}} e^{-\frac{\left|\mu_{i}\right|^{2}}{2 \sigma_{i}^{2}}+\frac{c_{i}}{2 \sigma_{i}^{2}}}\right. \\
&\left.\sum_{l=0}^{n_{0}} \frac{\Gamma\left(n_{0}+l\right) n_{0}^{1-2 l}}{\Gamma(l+1) \Gamma\left(n_{0}-l+1\right)}\left(\frac{c_{i}\left|\mu_{i}\right|^{2}}{4 \sigma_{i}^{4}}\right)^{l} \Gamma\left(-l, \frac{c_{i}}{2 \sigma_{i}^{2}}\right)\right] \\
& r_{2}=E\left\{\left(P_{M N 1}-r_{1 i}\right)^{2}\right\} \\
&=\sum_{i=1}^{I}\left[-\left(a_{i}-\frac{b_{i}}{c_{i}}-r_{1 i}\right)^{2}+\frac{\left(b_{i}-a_{i} c_{i}\right)^{2}}{2 \sigma_{i}^{2} c_{i}} e^{-\frac{\left|\mu_{i}\right|^{2}}{2 \sigma_{i}^{2}}}\right. \\
&\left.\sum_{l=0}^{n_{0}} \frac{\Gamma\left(n_{0}+l\right) n_{0}^{1-2 l}}{\Gamma(l+1) \Gamma\left(n_{0}-l+1\right)}\left(\frac{c_{i}\left|\mu_{i}\right|^{2}}{4 \sigma_{i}^{4}}\right)^{l} \Psi\left(l+1, l, \frac{c_{i}}{2 \sigma_{i}^{2}}\right)\right]
\end{aligned}
$$

where we have used (19), [27, eq. (3.383.10)] and [27, eq. (3.383.5)] in the derivation, and $\Psi(\cdot, \cdot, \cdot)$ is the confluent hypergeometric function [27]. We have found by trying several distributions that the Rician distribution gives a good approximation in this case. The Rician approximations to the PDF and $\mathrm{CDF}$ of $P_{M N 1}$ can be derived as

$$
\begin{aligned}
& f_{P_{M N 1}}(y) \approx \frac{y}{\sigma_{R}^{2}} e^{-\frac{y^{2}+v_{R}^{2}}{2 \sigma_{R}^{2}}} I_{0}\left(\frac{y v_{R}}{\sigma_{R}^{2}}\right) \\
& F_{P_{M N 1}}(y) \approx 1-Q_{1}\left(\frac{v_{R}}{\sigma_{R}}, \frac{y}{\sigma_{R}}\right)
\end{aligned}
$$




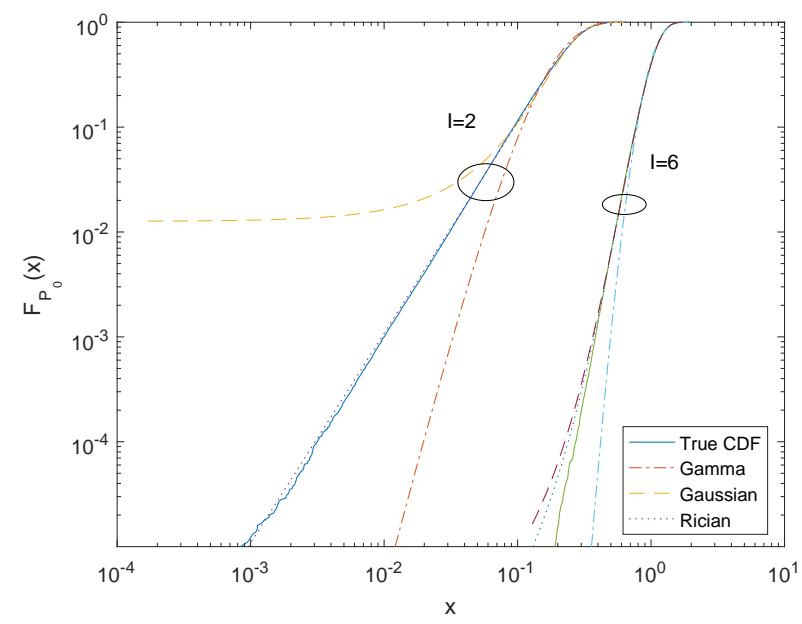

Figure 3. Comparison of different approximations to the CDF of $P_{M N 1}$ in (22).

where $v_{R}$ and $\sigma_{R}$ satisfy the moment-matching equations of $r_{1}=\sigma_{R} \sqrt{\frac{\pi}{2}} L_{\frac{1}{2}}\left(-\frac{v_{R}^{2}}{2 \sigma_{R}^{2}}\right)$ and $r_{2}=2 \sigma_{R}^{2}+v_{R}^{2}-\frac{\pi \sigma_{R}^{2}}{2} L_{\frac{1}{2}}^{2}\left(-\frac{v_{R}^{2}}{2 \sigma_{R}^{2}}\right)$, and they can be solved to give $v_{R}=\sqrt{r_{2}+r_{1}^{2}-\frac{4 r_{1}^{2}}{\pi L_{\frac{1}{2}}^{2}\left(-r_{0}\right)}}$ and $\sigma_{R}=\frac{r_{1}}{\sqrt{\frac{\pi}{2}} L_{\frac{1}{2}}\left(-r_{0}\right)}$, with $r_{0}$ being determined by the equation $\frac{r_{2}+r_{1}^{2}}{r_{1}^{2}}=\frac{4\left(1+r_{0}\right)}{\pi L_{\frac{1}{2}}\left(-r_{0}\right)}$, and $L_{i}(\cdot)$ is the Laguerre polynomial [27].

Fig. 3 compares different approximations to the CDF of the output power in (22). In the comparison, we set $x_{i}=1$, $2 \alpha_{i}^{2}=s_{i}=0.1 i \mathrm{~mW}, \beta^{2}=-40 \mathrm{dBm}$, and $a=2.463$, $b=1.635, c=0.826$ for the NLEH. One sees that the Rician approximation has the highest accuracy. Thus, in the following, we will use the Rician approximation for the output power of the NLEH model in (31) and (32).

\section{Special Case of Rayleigh Fading}

In this case, one has $s_{i}=0$ for $i=1,2, \cdots, I$. This will simplify some expressions.

Specifically, for the single harvester, the output power of the LEH model has

$$
\begin{aligned}
E\left\{P_{S L 1}\right\} & =\eta\left[2 \sum_{i=1}^{I}\left|x_{i}\right|^{2} \alpha_{i}^{2}+2 \beta^{2}\right] \\
f_{P_{S L 1}}(y) & =\frac{1}{2 \eta \sigma^{2}} e^{-\frac{y}{2 \eta \sigma^{2}}} \\
F_{P_{S L 1}}(y) & =1-e^{-\frac{y}{2 \eta \sigma^{2}}}
\end{aligned}
$$

The output power of the NLEH model has

$$
\begin{aligned}
E\left\{P_{S N 1}\right\} & =a-\frac{b}{c}+\frac{b-a c}{2 \sigma^{2}} e^{\frac{c}{2 \sigma^{2}}}\left[-E i\left(-\frac{c}{2 \sigma^{2}}\right)\right] \\
f_{P_{S N 1}}(y) & =\frac{1}{2 \sigma^{2}} e^{-\frac{c y}{2 \eta \sigma^{2}\left(a-\frac{b}{c}-y\right)}} \frac{a c-b}{\left(a-\frac{b}{c}-y\right)^{2}} \\
F_{P_{S N 1}}(y) & =1-e^{-\frac{c y}{2 \sigma^{2}\left(a-\frac{b}{c}-y\right)}}
\end{aligned}
$$

where $\operatorname{Ei}(\cdot)$ is the exponential integral [25].
For the multiple harvesters, the output power of the LEH model has

$$
\begin{aligned}
& E\left\{P_{M L 1}\right\}=2 \sum_{i=1}^{I} \eta_{i}\left|x_{i}\right|^{2} \alpha_{i}^{2}+2 \sum_{i=1}^{I} \eta_{i} \beta^{2} \\
& f_{P_{M L 1}}(y)=\sum_{i=1}^{I} \prod_{j=1, j \neq i}^{I} \frac{1}{2 \eta_{i} \sigma_{i}^{2}-2 \eta_{j} \sigma_{j}^{2}} e^{-\frac{y}{2 \eta_{i} \sigma_{i}^{2}}} \\
& F_{P_{M L 1}}(y)=\sum_{i=1}^{I} \prod_{j=1, j \neq i}^{I} \frac{2 \eta_{i} \sigma_{i}^{2}}{2 \eta_{i} \sigma_{i}^{2}-2 \eta_{j} \sigma_{j}^{2}}\left[1-e^{-\frac{y}{2 \eta_{i} \sigma_{i}^{2}}}\right]
\end{aligned}
$$

where we have used the result on the sum of exponential random variables [30]. The output power of the NLEH model has

$$
\begin{aligned}
& E\left\{P_{M N 1}\right\}=\sum_{i=1}^{I}\left[a_{i}-\frac{b_{i}}{c_{i}}-\frac{\left(b_{i}-a_{i} c_{i}\right)}{2 \sigma_{i}^{2}} e^{\frac{c_{i}}{2 \sigma_{i}^{2}}} \operatorname{Ei}\left(-\frac{c_{i}}{2 \sigma_{i}^{2}}\right)\right] \\
& f_{P_{M N 1}}(y)=\frac{1}{2 \pi} \int_{-\infty}^{\infty} \prod_{i=1}^{I} \phi_{i}(j v) e^{-j v y} d v \\
& F_{P_{M N 1}}(y)=\frac{1}{2 \pi} \int_{-\infty}^{\infty} \prod_{i=1}^{I} \phi_{i}(j v) \frac{e^{-j v y}}{j v} d v
\end{aligned}
$$

respectively, where we have used the characteristic function method to derive the distribution of a sum of random variables and $\phi_{i}(j v)=$ $\frac{c_{i} a_{i}-b_{i}}{2 \sigma_{i}^{2}} e^{j v\left(a_{i}-\frac{b_{i}}{c_{i}}\right)+\frac{c_{i}}{2 \sigma_{i}^{2}}} \sum_{k=0}^{\infty}\left(\frac{c_{i} a_{i}-b_{i}}{2 \sigma_{i}^{2}}\right)^{k}(j v)^{k+1} \gamma(-k$

$\left.1, j v\left(a_{i}-\frac{b_{i}}{c_{i}}\right)\right)$ is derived by using the Taylor series expansion of the exponential function and the definition of lower incomplete Gamma function.

\section{Shadowed Fading Signals}

In this case, the wireless signals experience both fading and shadowing. This is the case when the power transfer distance is large such that the shadowing effect cannot be ignored, as the effects of fading and shadowing depend on the distance between the transmitter and the receiver [31]. A widely used model for shadowing is the lognormal distribution [10]. However, this model often leads to mathematical intractability due to the logarithm operation in the exponent. An alternative shadowing model that is mathematically more tractable is the Gamma distribution [32]. This model can lead to the generalized $\mathrm{K}$ distribution for the composite Gamma-Gamma channels [33]. We assume the Gamma model for shadowing in this paper. Then, the average fading power $\alpha_{i}^{2}$ follows a Gamma distribution with PDF

$$
f_{\alpha_{i}^{2}}(x)=\frac{1}{\Gamma\left(k_{i}\right) \theta_{i}^{k_{i}}} e^{-\frac{x}{\theta_{i}}}
$$

where $k_{i}$ and $\theta_{i}$ are the shape and scale parameters of $\alpha_{i}^{2}$, respectively.

\section{A. Single Harvester}

The results when the wireless signal only suffers from Rician fading have been derived in Section III. When the signals experience both Rician fading and Gamma shadowing, 


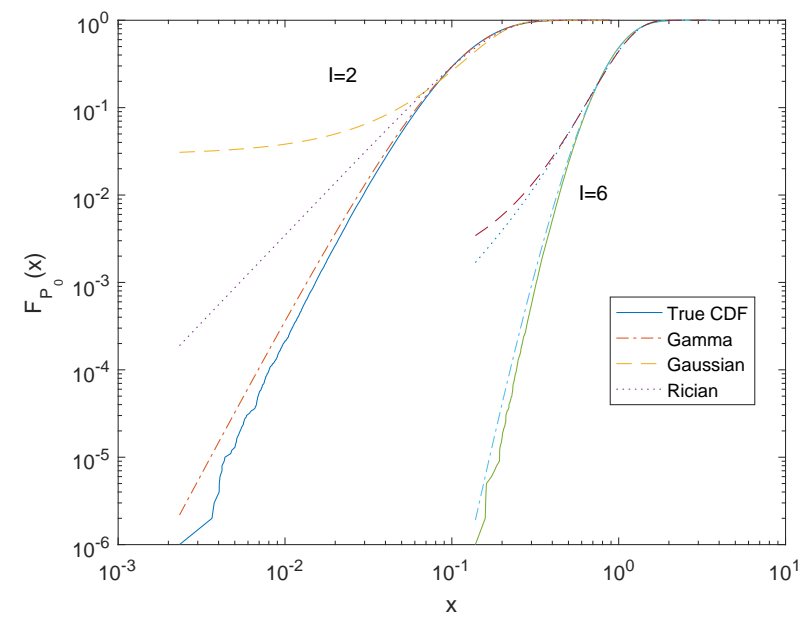

Figure 4. Comparison of different approximations to the CDF of $\sigma^{2}$ in (12).

we only need to average out the fading power in those expressions. To do this, the distribution of $\sigma^{2}$ is required. From (12), it is a weighted sum of $I$ Gamma random variables. The exact distribution of $\sigma^{2}$ can be derived by using the results in [34]. However, this expression is complicated. Thus, we resort to approximations. Fig. 4 compares different approximations to the distribution of $\sigma^{2}$ using moment-matching. In the figure, we set $x_{i}=1, k_{i}=2, \alpha_{i}^{2}=0.05 i m W, \theta_{i}=\alpha_{i}^{2} / k_{i}$ and $\beta^{2}=-40 \mathrm{dBm}$. One sees that the Gamma approximation has the highest accuracy. Thus, we use the Gamma approximation in the following as

$$
f_{\sigma^{2}}(x) \approx \frac{1}{\Gamma\left(k_{\sigma}\right) \theta_{\sigma}^{k_{\sigma}}} x^{k_{\sigma}-1} e^{-\frac{x}{\theta_{\sigma}}}
$$

where $k_{\sigma}=\frac{d_{1}^{2}}{d_{2}}, \theta_{\sigma}=\frac{d_{2}}{d_{1}}$, and $d_{1}=\sum_{i=1}^{I}\left|x_{i}\right|^{2} k_{i} \theta_{i}+\beta^{2}$ and $d_{2}=\sum_{i=1}^{I}\left|x_{i}\right|^{4} k_{i} \theta_{i}^{2}$ are the mean and variance of $\sigma^{2}$, respectively.

Using (46), the PDF and CDF for the output power of the LEH model can be derived as

$$
\begin{aligned}
& f_{P_{S L 2}}(y) \approx \sum_{l=0}^{n_{0}} \frac{\eta \Gamma\left(n_{0}+l\right) n_{0}^{1-2 l}\left(\frac{y|\mu|^{2}}{4 \eta}\right)^{l}}{\theta_{\sigma}^{k_{\sigma}} \Gamma\left(k_{\sigma}\right) \Gamma^{2}(l+1) \Gamma\left(n_{0}-l+1\right)} \\
& \left(\frac{\eta|\mu|^{2}+y}{2 \eta} \theta_{\sigma}\right)^{\frac{k_{\sigma}-2 l-1}{2}} K_{k_{\sigma}-2 l-1}\left(\sqrt{\frac{2 \eta|\mu|^{2}+2 y}{\eta \theta_{\sigma}}}\right) \\
& F_{P_{S L 2}}(y)=\int_{0}^{y} f_{P_{S L 2}}(t) d t
\end{aligned}
$$

where we have used the approximation in (19) followed by [27, eq. (3.471.9)], and $K_{k-2 l-1}(\cdot)$ is the $(k-2 l-1)$-th order modified Bessel function of the second type.

Similarly, the PDF and CDF for the NLEH model can be derived as

$$
\begin{aligned}
& f_{P_{S N 2}}(x) \approx \sum_{l=0}^{n_{0}} \frac{(a c-b) \Gamma\left(n_{0}+l\right) n_{0}^{1-2 l}\left(\frac{\left(c y|\mu|^{2} / 4\right)^{l}}{\left(a-\frac{b}{c}-y\right)^{l+2}}\right.}{\theta_{\sigma}^{k_{\sigma}} \Gamma\left(k_{\sigma}\right) \Gamma^{2}(l+1) \Gamma\left(n_{0}-l+1\right)} \\
& \quad\left(\frac{|\mu|^{2}+\frac{c y}{a-\frac{b}{c}-y}}{2} \theta_{\sigma}\right)^{\frac{k_{\sigma}-2 l-1}{2}} K_{k_{\sigma}-2 l-1}\left(\sqrt{\frac{2|\mu|^{2}+2 \frac{c y}{a-\frac{b}{c}-y}}{\theta_{\sigma}}}\right) \\
& F_{P_{S N 2}}(y)=\int_{0}^{y} f_{P_{S N 2}}(t) d t .
\end{aligned}
$$

The average harvested power for the LEH model can be derived from (17) as

$$
E\left\{P_{S L 1}\right\}=\eta\left[\left|\sum_{i=1}^{I} x_{i} s_{i}\right|^{2}+2 \sum_{i=1}^{I}\left|x_{i}\right|^{2} k_{i} \theta_{i}+2 \beta^{2}\right] .
$$

The average harvested power for the NLEH model can be derived as

$$
\begin{aligned}
& E\left\{P_{S N 2}\right\} \approx \sum_{l=0}^{n_{0}} \frac{\Gamma\left(n_{0}+l\right) n_{0}^{1-2 l}\left(\frac{|\mu|^{2}}{4}\right)^{l}}{\theta_{\sigma}^{k_{\sigma}} \Gamma\left(k_{\sigma}\right) \Gamma^{2}(l+1) \Gamma\left(n_{0}-l+1\right)} \\
& \int_{0}^{\infty}\left[\frac{a x+b}{x+c}-\frac{b}{c}\right] x^{l}\left(\frac{|\mu|^{2}+x}{2} \theta_{\sigma}\right)^{\frac{k_{\sigma}-2 l-1}{2}} \\
& K_{k_{\sigma}-2 l-1}\left(\sqrt{\frac{2|\mu|^{2}+2 x}{\theta_{\sigma}}}\right) d x .
\end{aligned}
$$

\section{B. Multiple Harvesters}

When multiple harvesters are used, we need to find the distribution of $\sigma_{i}^{2}$ first. Since $\sigma_{i}^{2}=\left|x_{i}\right|^{2} \alpha_{i}^{2}+\beta^{2}$, we approximate it as a Gamma distribution with PDF

$$
f_{\sigma_{i}^{2}}(x) \approx \frac{1}{\Gamma\left(p_{i}\right) q_{i}^{p_{i}}} x^{p_{i}-1} e^{-\frac{x}{q_{i}}}
$$

where $p_{i}=\frac{\left(\left|x_{i}\right|^{2} k_{i} \theta_{i}+\beta^{2}\right)^{2}}{\left|x_{i}\right|^{4} k_{i} \theta_{i}^{2}}$ and $q_{i}=\frac{\left|x_{i}\right|^{4} k_{i} \theta_{i}^{2}}{\left|x_{i}\right|^{2} k_{i} \theta_{i}+\beta^{2}}$. Using (24), the average harvested power for the LEH model can be derived as

$$
E\left\{P_{M L 2}\right\}=t_{1}=\sum_{i=1}^{I}\left[\eta_{i}\left|x_{i}\right|^{2}\left|s_{i}\right|^{2}+2 \eta_{i}\left|x_{i}\right|^{2} k_{i} \theta_{i}+2 \beta^{2} \eta_{i}\right]
$$

by averaging (24) over $\alpha_{i}^{2}$. Also, the average harvested power for the NLEH model can be derived as

$$
\begin{aligned}
E\left\{P_{M N 2}\right\} & =e_{1} \approx \sum_{i=1}^{I} e_{1 i}= \\
& \sum_{i=1}^{I} \sum_{l=0}^{n_{0}} \frac{\Gamma\left(n_{0}+l\right) n_{0}^{1-2 l}\left(\left|\mu_{i}\right|^{2} / 4\right)^{l}}{\Gamma^{2}(l+1) \Gamma\left(n_{0}-l+1\right) \Gamma\left(p_{i}\right) q_{i}^{p_{i}}} \\
& \int_{0}^{\infty}\left[\frac{a_{i} x+b_{i}}{x+c_{i}}-\frac{b_{i}}{c_{i}}\right] x^{l}\left(\frac{\left|\mu_{i}\right|^{2}+x}{2} q_{i}\right)^{\frac{p_{i}-2 l-1}{2}} \\
& K_{p_{i}-2 l-1}\left(2 \sqrt{\frac{\left|\mu_{i}\right|^{2}+x}{2 q_{i}}}\right) d t .
\end{aligned}
$$

The derivations of the exact PDF and CDF are challenging for both the LEH and NLEH models. We use momentmatching approximations. The means of $P_{M L 2}$ and $P_{M N 2}$ are 

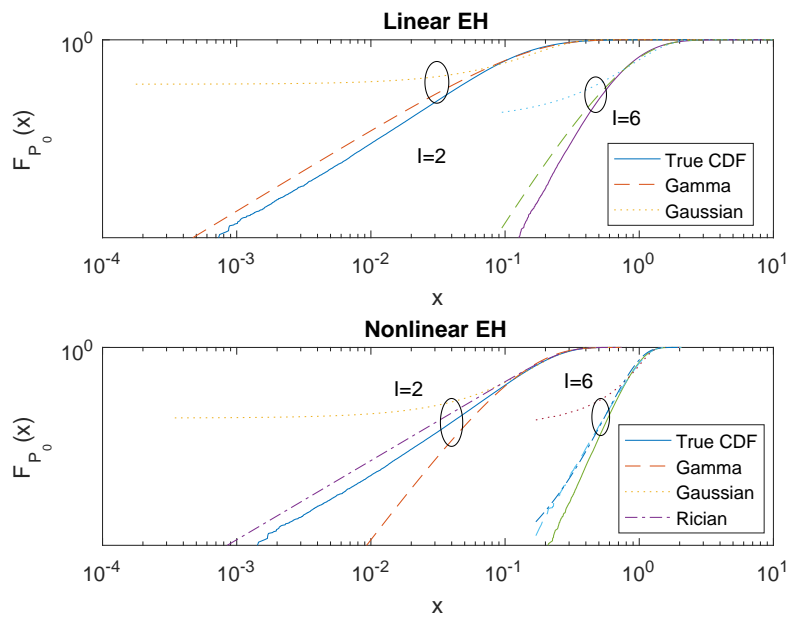

Figure 5. Comparison of different approximations to the CDF of the output power with both fading and shadowing.

given in (54) and (55), respectively. We need their variances too. They can be derived as

$$
\begin{aligned}
t_{2}=E & \left.E\left(P_{M L 2}-t_{1}\right)^{2}\right\}= \\
& \sum_{i=1}^{I}\left[4 \eta_{i}^{2}\left|x_{i}\right|^{2}\left|s_{i}\right|^{2} k_{i} \theta_{i}+4 \eta_{i} k_{i}^{2} \theta_{i}^{2}+4 \eta_{i} k_{i} \theta_{i}^{2}\right] \\
e_{2}=E & \left\{\left(P_{M N 2}-e_{1}\right)^{2}\right\} \approx \\
& \sum_{i=1}^{I} \sum_{l=0}^{n_{0}} \frac{\Gamma\left(n_{0}+l\right) n_{0}^{1-2 l}\left(\left|\mu_{i}\right|^{2} / 4\right)^{l}}{\Gamma^{2}(l+1) \Gamma\left(n_{0}-l+1\right) \Gamma\left(p_{i}\right) q_{i}^{p_{i}}} \\
& \int_{0}^{\infty}\left[\frac{a_{i} x+b_{i}}{x+c_{i}}-\frac{b_{i}}{c_{i}}-e_{1 i}\right]^{2} x^{l}\left(\frac{\left|\mu_{i}\right|^{2}+x}{2} q_{i}\right)^{\frac{p_{i}-2 l-1}{2}} \\
& K_{p_{i}-2 l-1}\left(2 \sqrt{\frac{\left|\mu_{i}\right|^{2}+x}{2 q_{i}}}\right) d t .
\end{aligned}
$$

Fig. 5 shows different approximations to the output power of the LEH and NLEH models when both Rician fading and Gamma shadowing occur. In the figure, we set $x_{i}=1, k_{i}=2$, $\alpha_{i}^{2}=0.05 i \mathrm{~mW}, \theta_{i}=\alpha_{i}^{2} / k_{i}$ and $\beta^{2}=-40 \mathrm{dBm}$. For the LEH model, the Gamma approximation has the highest accuracy, while for the NLEH model, the Rician approximation has the highest accuracy. Thus, we can approximate the PDF and $\mathrm{CDF}$ of $P_{M L 2}$ as

$$
\begin{aligned}
& f_{P_{M L 2}}(y) \approx \frac{1}{\Gamma\left(k_{M L 2}\right) \theta_{M L 2}^{k_{M L 2}}} x^{k_{M L 2}-1} e^{-\frac{y}{\theta_{M L 2}}} \\
& F_{P_{M L 2}}(y) \approx \frac{\gamma\left(k_{M L 2}, \frac{y}{\theta_{M L 2}}\right)}{\Gamma\left(k_{M L 2}\right)}
\end{aligned}
$$

where $k_{M L 2}=\frac{t_{1}^{2}}{t_{2}}, \theta_{M L 2}=\frac{t_{2}}{t_{1}}$ and $t_{1}$ and $t_{2}$ are given in (54) and (56), respectively. Similarly, the PDF and CDF of $P_{M N 2}$ can be approximated as

$$
\begin{aligned}
& f_{P_{M N 2}}(y) \approx \frac{y}{\sigma_{M N 2}^{2}} e^{-\frac{y^{2}+v_{M N 2}^{2}}{2 \sigma_{M N 2}^{2}}} I_{0}\left(\frac{y v_{M N 2}}{\sigma_{M N 2}^{2}}\right) \\
& F_{P_{M N 2}}(y) \approx 1-Q_{1}\left(\frac{v_{M N 2}}{\sigma_{M N 2}}, \frac{y}{\sigma_{M N 2}}\right)
\end{aligned}
$$

where the parameters of $v_{M N 2}$ and $\sigma_{M N 2}$ can be calculated in a way similar to those in (31) and (32), except that $r_{1}$ and $r_{2}$ are replaced by $e_{1}$ and $e_{2}$ respectively.

\section{Special Case of Rayleigh Fading}

In Rayleigh fading, some expressions in the previous two subsections can be simplified by setting $s_{i}=0$. Specifically, for the single harvester using the LEH model, one has

$$
\begin{aligned}
& E\left\{P_{S L 2}\right\}=2 \eta\left[\sum_{i=1}^{I}\left|x_{i}\right|^{2} k_{i} \theta_{i}+\beta^{2}\right] \\
& f_{P_{S L 2}}(y)=\frac{1}{\eta \theta_{\sigma}^{\frac{k_{\sigma}+1}{2}} \Gamma\left(k_{\sigma}\right)}\left(\frac{y}{2 \eta}\right)^{\frac{k_{\sigma}-1}{2}} K_{k_{\sigma}-1}\left(2 \sqrt{\frac{y}{2 \eta \theta_{\sigma}}}\right) \\
& F_{P_{S L 2}}(y)=\frac{\sqrt{2 \pi y} \theta_{\sigma}^{k_{\sigma}-\frac{1}{2}}}{\sqrt{\eta} \Gamma\left(k_{\sigma}\right)} \Gamma\left(2 k_{\sigma}-\frac{1}{2}\right)\left[K_{2 k_{\sigma}-1}\left(\sqrt{\frac{2 y}{\eta \theta_{\sigma}}}\right)\right. \\
&\left.L_{2 k_{\sigma}-2}\left(\sqrt{\frac{2 y}{\eta \theta_{\sigma}}}\right)+K_{2 k_{\sigma}-2}\left(\sqrt{\frac{2 y}{\eta \theta_{\sigma}}}\right) L_{2 k_{\sigma}-1}\left(\sqrt{\frac{2 y}{\eta \theta_{\sigma}}}\right)\right]
\end{aligned}
$$

where the first equation is obtained by letting $s_{i}=0$ in (51), the second equation is obtained by letting $\mu=0$, solving the integral using [27, eq. (3.471.9)] and using the transformation $y=\eta x$, while the last equation is obtained by integrating the second equation from 0 to $y$ and solving the integration using [27, eq. (6.561.4)], and $L_{v}(\cdot)$ is the modified Struve function of the $v$-th order [27].

For the single harvester using the NLEH model, one has

$$
\begin{gathered}
E\left\{P_{S N 2}\right\}=a-\frac{b}{c}+\frac{b-a c}{\theta_{\sigma} / 2}\left(\sqrt{\frac{2 c}{\theta_{\sigma}}}\right)^{k-1} S_{-k_{\sigma}, k_{\sigma}-1}\left(\sqrt{\frac{2 c}{\theta_{\sigma}}}\right) \\
f_{P_{S N 2}}(y)=\frac{1}{\theta_{\sigma}^{\frac{k_{\sigma}+1}{2}} \Gamma\left(k_{\sigma}\right)}\left(\frac{c y}{2\left(a-\frac{b}{c}-y\right)}\right)^{\frac{k_{\sigma}-1}{2}} \\
K_{k_{\sigma}-1}\left(2 \sqrt{\frac{c y}{2 \theta\left(a-\frac{b}{c}-y\right)}}\right) \frac{a c-b}{\left(a-\frac{b}{c}-y\right)^{2}} \\
F_{P_{S N 2}}(y)=\frac{\sqrt{\left.\frac{\sqrt{2 \pi c y} \theta_{\sigma}^{k_{\sigma}}-\frac{1}{2}}{\sqrt{a-\frac{b}{c}-y \Gamma\left(k_{\sigma}\right.}}\right)} \Gamma\left(2 k_{\sigma}-\frac{1}{2}\right)}{\left[K_{2 k_{\sigma}-1}\left(\sqrt{\frac{2 c y}{\theta_{\sigma}\left(a-\frac{b}{c}-y\right)}}\right) \cdot L_{2 k_{\sigma}-2}\left(\sqrt{\frac{2 c y}{\theta_{\sigma}\left(a-\frac{b}{c}-y\right)}}\right)\right.} \\
\left.+K_{2 k_{\sigma}-2}\left(\sqrt{\frac{2 c y}{\theta_{\sigma}\left(a-\frac{b}{c}-y\right)}}\right) L_{2 k_{\sigma}-1}\left(\sqrt{\frac{2 c y}{\theta_{\sigma}\left(a-\frac{b}{c}-y\right)}}\right)\right](67)
\end{gathered}
$$

where the first equation is obtained by letting $\mu=0$ in (52) and solving the integral using [27, eq. (6.565.7)], $S_{-k, k-1}(\cdot)$ is the Lommel function [27], and the second equation and the third equation are obtained in a similar way to those in (63) and (64).

For the case when multiple harvesters are used, one has the 
results for the LEH model as

$$
\begin{aligned}
& E\left\{P_{M L 2}\right\}=\sum_{i=1}^{I}\left[2 \eta_{i}\left|x_{i}\right|^{2} k_{i} \theta_{i}+2 \beta^{2} \eta_{i}\right] \\
& f_{P_{M L 2}}(y)=\frac{1}{2 \pi} \int_{-\infty}^{\infty} \prod_{i=1}^{I} \phi_{i}(j v) e^{-j v y} d v \\
& F_{P_{M L 2}}(y)=\frac{1}{2 \pi} \int_{-\infty}^{\infty} \prod_{i=1}^{I} \phi_{i}(j v) \frac{e^{-j v y}}{j v} d v
\end{aligned}
$$

where the first equation is obtained by setting $s_{i}=0$ in (54), the characteristic function is derived as $\phi_{i}(j v)=$ $\frac{e^{\frac{1}{4 j v \eta \theta_{i}}}}{\left(2 j v \eta \theta_{i}\right)^{k_{i} / 2}} W_{-\frac{k_{i}}{2},-\frac{k_{i}-1}{2}}\left(\frac{1}{2 j v \eta \theta_{i}}\right)$ by using the definition and [27, eq. (6.643.6)], and $W_{-\frac{k_{i}}{2},-\frac{k_{i}-1}{2}}(\cdot)$ is the Whittaker function.

Similarly, for multiple harvesters, the results for the NLEH model are given by

$$
\begin{gathered}
E\left\{P_{M N 2}\right\}=\sum_{i=1}^{I}\left[a_{i}-\frac{b_{i}}{c_{i}}+\frac{b_{i}-a_{i} c_{i}}{\theta_{i} / 2}\left(\sqrt{\frac{2 c_{i}}{\theta_{i}}}\right)^{k_{i}-1}\right. \\
\left.S_{-k_{i}, k_{i}-1}\left(\sqrt{\frac{2 c_{i}}{\theta_{i}}}\right)\right] \\
f_{P_{M N 2}}(y)=\frac{1}{2 \pi} \int_{-\infty}^{\infty} \prod_{i=1}^{I} \phi_{i}(j v) e^{-j v y} d v \\
F_{P_{M N 2}}(y)=\frac{1}{2 \pi} \int_{-\infty}^{\infty} \prod_{i=1}^{I} \phi_{i}(j v) \frac{e^{-j v y}}{j v} d v
\end{gathered}
$$

where the first equation is obtained by setting $\mu_{i}=0$ in (55) and solving the integral using [27, 6.565.7], and $\phi_{i}(j v)=$ $\frac{e^{j v\left(a_{i}-\frac{b_{i}}{c_{i}}\right)}}{\theta_{i}^{\frac{k_{i}+1}{2}} \Gamma\left(k_{i}\right)} \int_{0}^{\infty} e^{j v \frac{b_{i}-a_{i} c_{i}}{x+c_{i}}}(x / 2)^{\frac{k_{i}-1}{2}} K_{k_{i}-1}\left(2 \sqrt{\frac{x}{2 \theta_{i}}}\right) d x$. The analytical expressions for the NLEH model are generally more complicated than those for the LEH model. They can be further simplified for some special cases. For example, one has $f(x)=\frac{a x+b}{x+c}-\frac{b}{c}=a-\frac{b}{c}+\frac{b-a c}{x+c}$. When the input power $x$ is very small, using the Taylor series expansion of $\frac{1}{1+x}$ and ignoring the higher order terms, one has $\frac{1}{1+x} \approx 1-x$. One can derive $f(x) \approx \frac{a c-b}{c^{2}} x$. Thus, the expressions for the NLEH model can be simplified by letting $\eta=\frac{a c-b}{c^{2}}$ in those for the LEH model, when the input power is very small. Since these results are quite straightforward, they are not presented here to make the paper compact. From the system design's point of view, the NLEH model is similar to the LEH model or the practical harvester has a linear range around $x=0$, with an equivalent conversion efficiency of $\eta=\frac{a c-b}{c^{2}}$.

Note that the above results include the noise power $2 \beta^{2}$ in the harvested power. In most literature on energy harvesting (see [36] and references citing it), the noise as a source of energy is not considered. This is because these references have assumed that the noise is negligible, such as equation (13) in [36]. Without this assumption, the noise energy can be harvested in the same way as the transmitted energy. Physically, the noise cannot be removed from the transmitted energy and hence will be harvested along with the transmitted energy from the received signal. Mathematically, if the square of the received signal is ergodic, its statistical average can be approximated by its temporal average. Since its statistical average includes the noise variance and its temporal average is actually the power, the harvested power will include the noise variance. Our results are general enough to include [36] as a special case when $2 \beta^{2}$ equals 0 or is small.

\section{Outage And Power Transfer Strategy}

\section{A. Outage Probability}

The first application of the preceding results is the calculation of the probability of power outage. For energy harvesting communications, since the harvested power is a random variable, it is possible that the available power may drop below the required power, causing a power outage. This probability is defined as $\operatorname{Pr}\left\{P_{o}<T_{0}\right\}$, where $P_{o}$ is the harvested power in (3) or (6) and $T_{0}$ is the required transmission power. Thus, we can use the CDF derived in the previous two sections directly to calculate the power outage probability.

Also, using the expression of outage and the CDF derived, useful insights for practical system designs can be obtained. For example, from (14) and (16), when a single harvester is used for Rician faded signals, the outage decreases when $\sigma$ increases. Thus, from (12), one must increase $I,\left|x_{i}\right|^{2}$ or $\alpha_{i}^{2}$ in the system to reduce outage. Also, increasing $\eta$ for LEH and $(a c-b) / c^{2}$ for NLEH can reduce the outage. From (29), when multiple linear harvesters are used for Rician faded signals, the outage decreases when $m_{1}$ increases. Thus, from (27), one can increase $I, \eta_{i}, \sigma_{i}^{2},\left|x_{i}\right|^{2}$ or $\left|s_{i}\right|^{2}$ to reduce the outage. Similarly, from (59), when multiple linear harvesters are used for Gamma-shadowed Rician fading signals, the outage decreases when $t_{1}$ increases. Thus, the outage of the system can be reduced by increasing $I, \eta_{i},\left|x_{i}\right|^{2},\left|s_{i}\right|^{2}, k_{i}$, or $\theta_{i}$ from (54).

\section{B. Power Transfer Strategy}

The second application of our results is to find the optimal power transfer strategy that maximizes the average harvested power, subject to the constraint that the total transferred power is fixed for all sources. Mathematically, the optimization problem is given by

$\left\{\hat{x}_{1}, \cdots, \hat{x}_{I}\right\}=\max _{x_{1}, \cdots, x_{I}}\left\{E\left\{P_{o}\right\}\right\}$, where $\sum_{i=1}^{I}\left|x_{i}\right|^{2}=P$.

This can only be solved for the LEH model and the NLEH model with Rayleigh fading. For the NLEH model with general Rician fading, exhaustive search has to be performed to find the optimum values.

1) LEH model with single harvester: For the LEH model using a single harvester, when there is only Rician fading, one has the average harvested power in (17), which can be rewritten as

$$
\begin{aligned}
& E\left\{P_{S L 1}\right\}=\eta\left[\sum_{i=1}^{I}\left(\epsilon_{i}^{2}+2 \alpha_{i}^{2}\right) \omega_{i}^{2}+2 \sum_{i=1}^{I} \sum_{j=1}^{i-1}\right. \\
&\left.\omega_{i} \omega_{j} \epsilon_{i} \epsilon_{j} \cos \left(\psi_{i}+\phi_{i}-\psi_{j}-\phi_{j}\right)+2 \beta^{2}\right]
\end{aligned}
$$


where $\omega_{i}, \psi_{i}, \epsilon$ and $\phi_{i}$ are the magnitude and phase of the transmitting waveform, the magnitude and phase of the lineof-sight in Rician channels, respectively. Since $\cos (\theta) \leq 1$, the optimum values of $\psi_{i}$ satisfies $\cos \left(\psi_{i}+\phi_{i}-\psi_{j}-\phi_{j}\right)=1$, or $\psi_{i}=-\phi_{i}$ for $i=1,2, \cdots, I$. In this case, one has

$$
\begin{aligned}
J & =\eta\left[\sum_{i=1}^{I}\left(\epsilon_{i}^{2}+2 \alpha_{i}^{2}\right) \omega_{i}^{2}+2 \sum_{i=1}^{I} \sum_{j=1}^{i-1} \omega_{i} \omega_{j} \epsilon_{i} \epsilon_{j}+2 \beta^{2}\right] \\
& =\eta\left[\omega \Delta \omega^{T}+2 \beta^{2}\right]
\end{aligned}
$$

where $\omega=\left[\begin{array}{llll}\omega_{1} & \omega_{2} & \cdots & \omega_{I}\end{array}\right], \boldsymbol{\Delta}_{i j}=\epsilon_{i} \epsilon_{j}$ when $i \neq j$ and $\boldsymbol{\Delta}_{i j}=\epsilon_{i}^{2}+2 \alpha_{i}^{2}$ when $i=j$. Since $\boldsymbol{\Delta}$ is a real symmetric matrix, and the constraint $\sum_{i=1}^{I}\left|x_{i}\right|^{2}=P$ is equivalent to $\omega \mathbf{B} \omega^{T}=1$, where $\mathbf{B}$ is a real diagonal matrix with $\frac{1}{P}$ on the diagonal lines, according to [35], the optimum values of $\omega_{i}$ are the eigenvector corresponding to the largest eigenvalue of $\mathbf{B}^{-1} \boldsymbol{\Delta}$.

Theorem 5.1: For the LEH model using a single harvester in Rician fading, the maximum average harvested power is $\eta\left(\lambda_{\max }+2 \beta^{2}\right)$, where $\lambda_{\max }$ is the largest eigenvalue of $\mathbf{B}^{-1} \boldsymbol{\Delta}$. The optimum phases of the waveforms are $\psi_{i}=-\phi_{i}$, and the optimum magnitudes of the waveforms are $\omega=\mathbf{v}_{\max }$, where $\mathbf{v}_{\max }$ is the eigenvector corresponding to the largest eigenvalue of $\mathbf{B}^{-1} \boldsymbol{\Delta}$.

In the special case when there is only Rayleigh fading, $s_{i}=$ 0 . The average harvested power using a single harvester with Rayleigh fading only is derived from (75) as $E\left\{P_{S L 1}\right\}=$ $\eta\left[\sum_{i=1}^{I}\left(2 \alpha_{i}^{2}\right) \omega_{i}^{2}+2 \beta^{2}\right]$. Thus, the maximum average harvested power is given by $2 \eta P \alpha_{\hat{i}}^{2}+2 \beta^{2}$, where $\hat{i}=\max _{i=1,2, \cdots, I}\left\{2 \alpha_{i}^{2}\right\}$ and which is achieved by letting $\omega_{i}^{2}=P$ when $i=\hat{i}$ and $\omega_{i}^{2}=0$ when $i \neq \hat{i}$. Thus, in Rayleigh fading channels, the best power transfer strategy is always to transmit the full power at the source with the best channel condition and switch off all other sources.

In the case when both Rician (or Rayleigh) fading and Gamma shadowing occur, similar results can be obtained, except that $\alpha_{i}^{2}$ should be replaced by $k_{i} \theta_{i}$ in the matrix $\Delta$.

2) LEH model with multiple harvesters: For the LEH model using multiple harvesters, when there is only Rician fading, the average harvester power is given by (24) to give

$$
\begin{aligned}
E\left\{P_{M L 1}\right\} & =\sum_{i=1}^{I} \eta_{i}\left(\epsilon_{i}^{2}+2 \alpha_{i}^{2}\right) \omega_{i}^{2}+2 \sum_{i=1}^{I} \eta_{i} \beta^{2} \\
& =\omega \mathbf{A} \omega^{T}+2 \sum_{i=1}^{I} \eta_{i} \beta^{2}
\end{aligned}
$$

where $\mathbf{A}$ is a diagonal matrix with the $i$-th element on the diagonal line given by $\mathbf{A}(i, i)=\eta_{i}\left(\epsilon_{i}^{2}+2 \alpha_{i}^{2}\right)$. Since $\mathbf{B}^{-1}$ is also a diagonal matrix with $P$ on the diagonal line, the index of the largest eigenvalue of $\mathbf{B}^{-1} \boldsymbol{\Delta}$ is actually given by $\hat{i}=\max _{i=1,2, \cdots, I}\left\{\eta_{i}\left(\epsilon_{i}^{2}+2 \alpha_{i}^{2}\right)\right\}$. The eigenvector corresponding to the largest eigenvalue is $\left[\begin{array}{llllll}0 & 0 & \cdots & \sqrt{P} & \cdots & 0\end{array}\right]$, where the $\hat{i}$-th element is the only non-zero element.

Theorem 5.2: For the LEH model using multiple harvesters in Rician fading channels, the maximum average harvested power is $\eta_{\hat{i}}\left(\epsilon_{\hat{i}}^{2}+2 \alpha_{\hat{i}}^{2}\right) P+2 \sum_{i=1}^{I} \eta_{i} \beta^{2}$, where $\hat{i}=\max _{i=1,2, \cdots, I}\left\{\eta_{i}\left(\epsilon_{i}^{2}+2 \alpha_{i}^{2}\right)\right\}$. The optimum magnitudes of the waveforms are $\omega_{i}^{2}=P$, when $i=\hat{i}$, and $\omega_{i}^{2}=0$, when $i \neq \hat{i}$. The phases of the waveforms do not affect the harvested power.

In the special case of Rayleigh fading, one has from (77) $E\left\{P_{M L 1}\right\}=2 \sum_{i=1}^{I} \eta_{i} \alpha_{i}^{2} \omega_{i}^{2}+2 \sum_{i=1}^{I} \eta_{i} \beta^{2}$. Thus, the maximum average harvested power is given by $2 \eta_{\hat{i}} P \alpha_{\hat{i}}^{2}+$ $2 \sum_{i=1}^{I} \eta_{i} \beta^{2}$, where $\hat{i}=\max _{i=1,2, \cdots, I}\left\{2 \eta_{i} \alpha_{i}^{2}\right\}$ and which is achieved by letting $\omega_{i}^{2}=P$ when $i=\hat{i}$ and $\omega_{i}^{2}=0$ when $i \neq \hat{i}$.

When there are both Rician fading and Gamma shadowing, similar results apply, except that $\alpha_{i}^{2}$ are replaced by $k_{i} \theta_{i}$ in A.

3) NLEH model with single harvester: When the NLEH model is used with a single harvester in Rayleigh fading channels, the average harvested power is given by (36). Denote $g(x)=x e^{x}[-E i(-x)]$. It can be shown that $g(x)$ is a monotonically increasing function of $x$. Thus, the maximization of the average harvested power in (36) is equivalent to the maximization of $2 \sigma^{2}$. Since one has (12), which is again a quadratic form of $\omega_{i}$, one sees that the results for the LEH model in Rayleigh fading can be applied here.

Theorem 5.3: For the NLEH model using a single harvester in Rayleigh fading channels, the maximum average harvested power is given by $a-\frac{b}{c}+\frac{b-a c}{2 \hat{\sigma}^{2}} e^{\frac{c}{2 \hat{\sigma}^{2}}}\left[-E i\left(-\frac{c}{2 \hat{\sigma}^{2}}\right)\right]$, where $2 \hat{\sigma}^{2}=2 P \alpha_{\hat{i}}^{2}+2 \beta^{2}$ and $\hat{i}=\max _{i=1,2, \cdots, I}\left\{2 \alpha_{i}^{2}\right\}$. The optimum magnitudes are $\omega_{i}^{2}=P$ when $i=\hat{i}$ and $\omega_{i}^{2}=0$ when $i \neq \hat{i}$.

The case with general Rician fading cannot be solved and its optimum values have to be found using exhaustive search.

4) NLEH model with multiple harvesters: When the NLEH model is used with multiple harvesters in Rayleigh fading channels, the average harvested power is given by (42). We can use the Lagrange multiplier to find the optimum solution. First, the function $g(x)$ can be curve-fitted as $g(x) \approx \frac{0.98 x+0.12}{x+0.86}$ for $0<x<30$. Then, one needs to maximize the objective function

$$
\begin{aligned}
W= & \sum_{i=1}^{I}\left(a_{i}-\frac{b_{i}}{c_{i}}\right)+\sum_{i=1}^{I}\left(b_{i}-a_{i} c_{i}\right) \frac{0.98 c_{i}+0.12\left(\omega_{i}^{2} 2 \alpha_{i}^{2}+2 \beta^{2}\right)}{c_{i}+0.86\left(\omega_{i}^{2} 2 \alpha_{i}^{2}+2 \beta^{2}\right)} \\
& +\lambda\left(P-\sum_{i=1}^{I} \omega_{i}^{2}\right)
\end{aligned}
$$

where $\lambda$ is the Lagrange multiplier and we have used the approximate $g(x)$ and the expression of $2 \sigma_{i}^{2}$ in (42). Using (78), the optimum values can be derived as

$$
\omega_{i}^{2}=\frac{\sqrt{1.2 \alpha_{i}^{2} c_{i}\left(a_{i} c_{i}-b_{i}\right) / \lambda_{0}}-c_{i}-1.72 \beta^{2}}{1.72 \alpha_{i}^{2}}
$$

with $\lambda_{0}=\left(\frac{\sum_{i=1}^{I} \frac{\sqrt{1.2 \alpha_{i}^{2} c_{i}\left(a_{i} c_{i}-b_{i}\right)}}{1.72 \alpha_{i}^{2}}}{P+\sum_{i=1}^{I} \frac{c_{i}+1.72 \beta^{2}}{1.72 \alpha_{i}^{2}}}\right)^{2}$. Again, for the general case of Rician fading, their optimum values have to be found by exhaustive search. Some numerical examples will be shown in the next section. 


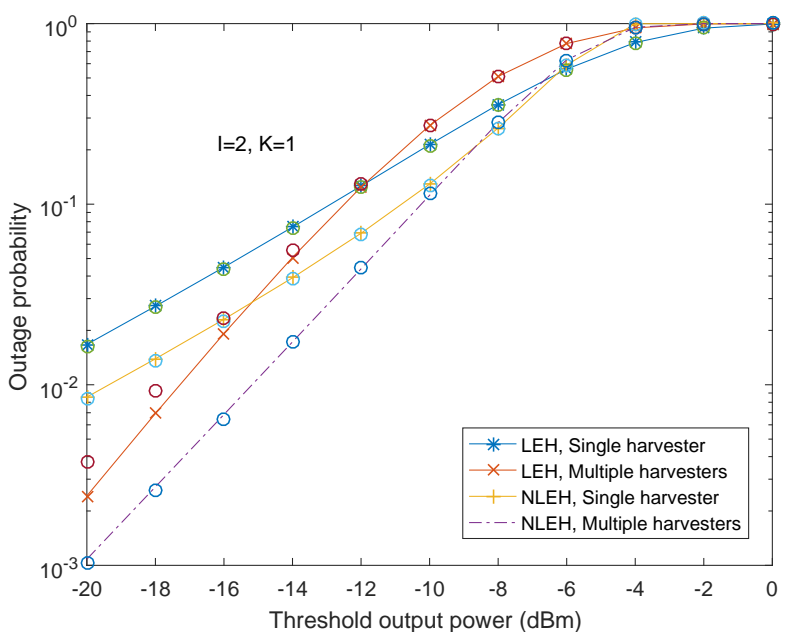

Figure 6. Outage probability vs. $T_{0}$ for $I=2$ and $K=1$ when there is Rician fading.

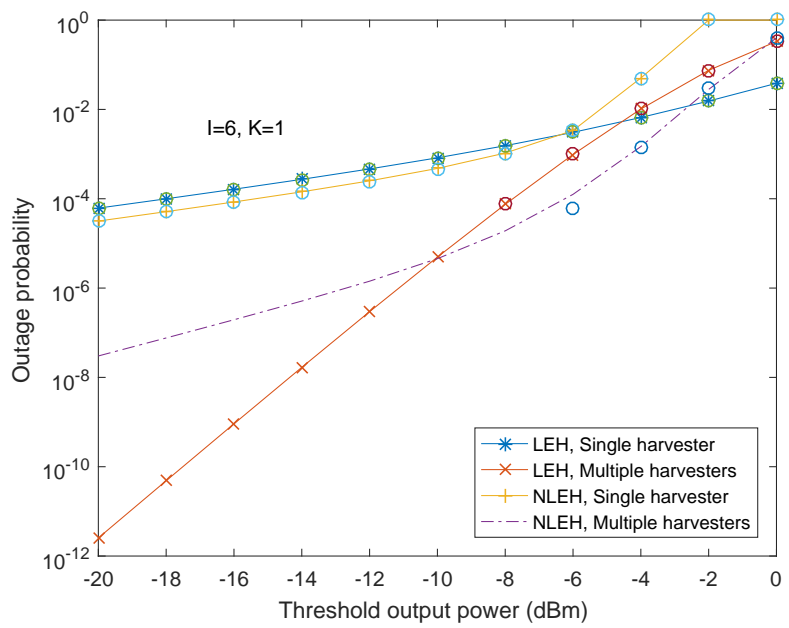

Figure 7. Outage probability vs. $T_{0}$ for $I=6$ and $K=1$ when there is Rician fading.

\section{Numerical Results And Discussion}

In this section, some numerical examples are given to show the power outage probability under different conditions and the optimal power transfer strategy for specific system settings.

Figs. 6 and 7 show the outage probability vs. $T_{0}$ when there is only Rician fading. In these figures, $x_{i}=1, K=\frac{\left|s_{i}\right|^{2}}{2 \alpha_{i}^{2}}=1$ is the Rician $\mathrm{K}$ factor of the $i$-th channel, $\alpha_{i}^{2}=0.05 i m W$, $\beta^{2}=-40 \mathrm{dBm}, \eta=0.3$ for the LEH model and $a=2.463$, $b=1.635, c=0.826$ for the NLEH model. Several observations can be made from Fig. 6. Firstly, for the LEH model, the use of a single harvester has a smaller outage probability than the use of multiple harvesters when $T_{0}>-12 \mathrm{dBm}$, indicating that one should use a single harvester when the required transmission power is large. This is explained as follows. Comparing (2) with (5), one sees that the input powers of multiple harvesters only contain the squared terms of the input power of a single harvester. This can also be seen by comparing $E\left\{P_{S L 1}\right\}$ and $E\left\{P_{M L 1}\right\}$ and assuming negligible noise. Thus, a single harvester harvests more power

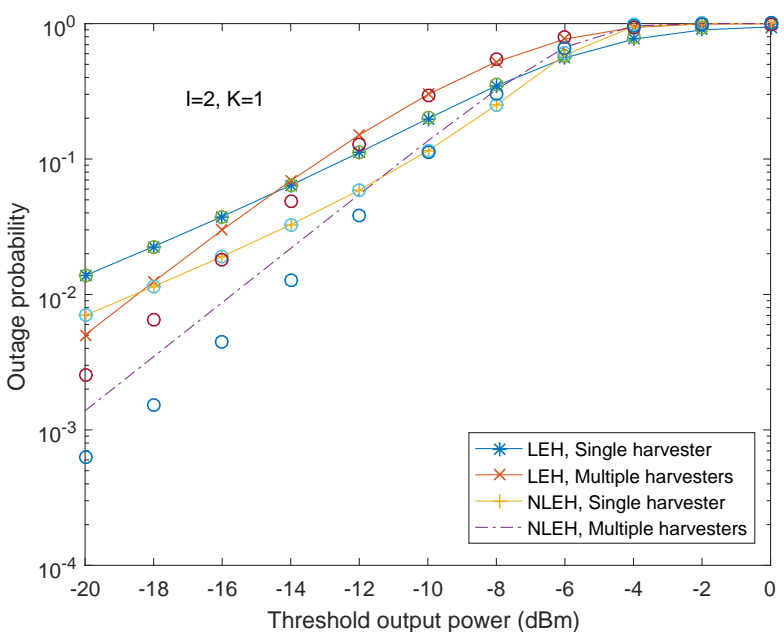

Figure 8. Outage probability vs. $T_{0}$ for $I=2$ and $K=1$ when there are both Rician fading and Gamma shadowing.

to give a smaller outage. Similarly, for the NLEH model, when $T_{0}>-8 \mathrm{dBm}$, it is better to use a single harvester. Secondly, comparing LEH and NLEH models, the LEH model has a smaller outage probability when $T_{0}>-6 \mathrm{dBm}$ and a larger outage when $T_{0}<-6 \mathrm{dBm}$, for a single harvester. For multiple harvesters, the LEH model always has a larger outage probability. This implies that the NLEH model has advantage for multiple harvesters or for smaller transmission power. This is because the NLEH model suffers from nonlinear saturation when the input power is too large so that it is advantageous to split the total power into several smaller signals or use a smaller transmission power. Fig. 7 shows the outage at $I=6$. One sees that the outage probability has decreased in all cases, because more sources lead to more harvested power. Also, for multiple harvesters, the curve for the LEH model crosses with the curve for the NLEH model when $T_{0}=0 \mathrm{dBm}$. When the threshold is large and increases, the NLEH model cannot provide enough harvested power and hence, its outage approaches the limit of $100 \%$ outage in a much faster rate than the LEH model. Consequently, the two curves cross.

Fig. 8 shows the outage probability vs. $T_{0}$ when both Rician fading and Gamma shadowing occur. In this figure, $x_{i}=1, K=\frac{\left|s_{i}\right|^{2}}{2 \alpha_{i}^{2}}=1$ is the Rician $\mathrm{K}$ factor of the $i$-th channel, $\alpha_{i}^{2}=0.05 i m W, k_{i}=2, \theta_{i}=\alpha_{i}^{2} / k_{i}$, $\beta^{2}=-40 \mathrm{dBm}, n_{0}=20, \eta=0.3$ for the LEH model and $a=2.463, b=1.635, c=0.826$ for the NLEH model. Similar observations can be made. These results give very useful design guidelines for wireless power transfer. For example, if the required transmission power of the portable device is large, one should use a single harvester or a practical nonlinear energy harvester with a linear range as large as possible. Otherwise, one should use multiple harvesters or the nonlinear range of the practical energy harvesters. Also, simulation results for outage above $10^{-6}$ (outage below $10^{-6}$ requires an excessively long time and hence is not simulated) are shown using the circle markers in Figs. 6 - 8. One sees that the simulation matches well with the analysis in most cases. When the threshold is smaller than $-12 \mathrm{dBm}$, there is 
a noticeable difference between simulation and analysis for multiple harvesters in Fig. 8. This agrees with the observations from Fig. 5. Practical values of the required transmission power are often larger than $-12 \mathrm{dBm}$ so that this will not cause problems.

Table I shows the maximum average harvested power in $\mathrm{mW}$ followed by the optimum magnitudes for $\left(\omega_{1}\right)$ when two sources transmit and $\left(\omega_{1}, \omega_{2}\right)$ when three sources transmit, obtained by exhaustive search. We set $k_{i}=2, \alpha_{i}^{2}=0.05 i \mathrm{~mW}$, $\theta_{i}=\alpha_{i}^{2} / k_{i}, \beta^{2}=-40 \mathrm{dBm}, \eta=0.3, a=2.463, b=1.635$, $c=0.826$ in the search.

One sees from Table I that the average harvested power increases with $I$, as expected, as more sources lead to more harvested power. Also, shadowing slightly reduces the average harvested power, except for LEH, as we set $\alpha_{i}^{2}=k_{i} \theta_{i}$ in the search. Also, the maximum average harvested power using a single harvester is always larger than that using multiple harvesters, as the input power of a single harvester is larger than the sum of those of multiple harvesters. It can be shown that these values are the same as those predicted by the theorems derived above, when available.

\section{CONCLUSION}

In this paper, we have analyzed the power harvested from multiple wireless signals experiencing either Rician fading or Gamma-shadowed Rician fading. Both the LEH and NLEH models have been studied. The analytical expressions for the average harvested power, the PDF and CDF have been derived. Using these expressions, the power outage probability has been calculated and the optimal power transfer strategy has been discussed. Numerical results have shown that, when the required transmission power of the harvesting device is large, it is favorable to use the linear range of a practical nonlinear harvester or a single harvester. Also, for Rayleigh fading channels, the optimal power strategy is to put the total power in the source with the best channel condition and switch off all other sources, while for general Rician fading channels, the optimal strategy can be obtained by choosing the magnitude and the phase of the transmitting waveform based on the channel parameters.

\section{REFERENCES}

[1] M. Pinuela, P.D. Mitcheson, and S. Lucyszyn, "Ambient RF energy harvesting in urban and semi-urban environments," IEEE Trans. Microw. Theory Techn., vol. 61, no. 7, pp. 2715-2726, Jul. 2013.

[2] F. Azmat, Y. Chen, N. Stocks, "Predictive modelling of RF energy for wireless powered communications," IEEE Comm. Lett., vol. 20, pp. 173 - 176, Jan. 2016.

[3] Z. Xie, Y. Chen, Y. Gao, Y. Wang, Y. Su, "Wireless powered communication networks using peer harvesting," to appear in IEEE Access.

[4] P. He, L. Zhao, S. Zhou, Z. Niu, "Recursive water-filling for wireless links with energy harvesting transmitters," IEEE Trans. Veh. Technol., vol. 63, pp. 1232 - 1241, Mar. 2014.

[5] B. Medepally, N.B. Mehta, "Voluntary energy harvesting relays and selection in cooperative wireless networks," IEEE Trans. Wireless Commun., vol. 9, pp. 3543 - 3553, Nov. 2010 .

[6] W. Huang, H. Chen, Y. Li, B. Vucetic, "On the Performance of MultiAntenna Wireless-Powered Communications with Energy Beamforming", IEEE Trans. Veh. Technol., vol. 65, no. 3, pp. 1801 - 1808, Mar. 2016.

[7] K. Huang and V.K.N. Lau, "Enabling wireless power transfer in cellular networks: architecture, modeling and deployment," IEEE Trans. Wireless Commun., vol. 13, pp. 902 - 912, Feb. 2014.
[8] H. Ju, R. Zhang, "Throughput maximization in wireless powered communication networks," IEEE Trans. Wireless Commun., vol. 13, pp. 418 - 428, Jan. 2014.

[9] L.R.Varshney, "Transporting information and energy simultaneously," in Proc. IEEE Int. Symp. Inf. Theory, pp. 1612-1616, June 2008.

[10] G.L. Stuber, Principles of Mobile Communication, 2nd Ed. Kluwer: London, 2001.

[11] D. Altinel, G.K. Kurt, "Energy harvesting from multiple RF sources in wireless fading channels," IEEE Trans. Veh. Technol., vol. 65, pp. 8854 - 8864, Nov. 2016.

[12] T. Le, K. Mayaram, and T. Fiez, "Efficient far-field radio frequency energy harvesting for passively powered sensor networks," IEEE J. SolidState Circuits, vol. 43, pp. 1287-1302, May 2008.

[13] J. Guo and X. Zhu, "An improved analytical model for RF-DC conversion efficiency in microwave rectifiers," in Proc. IEEE MTT-S Int. Microw. Symp. Dig., Jun. 2012, pp. 1-3.

[14] E. Boshkovska, D.W.K. Ng, N. Zlatanov, R. Schober, "Practical nonlinear energy harvesting model and resource allocation for SWIPT systems," IEEE Comm. Lett., vol. 19, pp. 2082 - 2085, Dec. 2015.

[15] M. Stoopman, S. Keyrouz, H.J. Visser, K. Philips, W.A. Serdijin, "A self-calibrating RF energy harvester generating $1 \mathrm{~V}$ at $-26.3 \mathrm{dBm}, " 2013$ Symposium on VLSI Circuits (VLSIC), pp. C226 - C227, 2013.

[16] D. Yeager, F. Zhang, A. Zarrasvand, B.P. Otis, "A 9.2uA Gen 2 compatible UHF RFID sensing tag with $-12 \mathrm{dBm}$ sensitivity and $1.25 \mathrm{uVrms}$ input-referred noise floor," IEEE International Solid-State Circuits Conference Digest of Technical Papers (ISSCC) 2010, pp. 52-53, 2010.

[17] S. Scorcioni , L. Larcher, A. Bertacchini, "A reconfigurable differential CMOS RF energy scavenger with $60 \%$ peak efficiency and $-21 \mathrm{dBm}$ sensitivity," IEEE Microwave and Wireless Components Letters, vol. 23, pp. 155-157, 2013.

[18] H. Sun, Y. Guo, M. He, Z. Zhong, "A dual band rectenna using broadband Yagi antenna array for ambient RF power harvesting," IEEE Antennas and Wireless Propagation Letters, vol. 12, pp. 918-921, 2013.

[19] Y. Chen, K.S. Thomas, R.A.A. Abd, "New formula for conversion efficiency of RF EH and its wireless applications," IEEE Trans. on Veh. Technol., vol. 65, pp. 9410 - 9414, Nov. 2016.

[20] P. He, L. Zhao, S. Zhou, Z. Niu, "Optimal recursive power allocation for energy harvesting system with multiple antennas," IEEE Trans. Veh. Technol., vol. 64, pp. 4525 - 4536, Oct. 2015.

[21] Y. Chen, "Energy harvesting AF relaying in the presence of interference and Nakagami-m fading," IEEE Trans. on Wireless Commun., vol. 15, pp. 1008 - 1017, Feb. 2016.

[22] Y. Chen, D. Da Costa, H. Ding, "Effect of CCI on WPC With Time-Division Energy and Information Transmission," IEEE Wireless Commun. Lett., vol. 5, pp. 168 - 171, Feb. 2016.

[23] W. Feng, Y. Wang, N. Ge, J. Lu, and J. Zhang, "Virtual MIMO in multi-cell distributed antenna systems: coordinated transmissions with large-scale CSIT," IEEE J. on Selected Areas in Commun., vol. 31, pp. 2067-2081, Oct. 2013.

[24] J.G. Proakis, Digital Communications, 4th Ed. McGraw-Hill: Singapore, 2001.

[25] M. Abramowitz, I.A. Stegun, Handbook of Mathematical Functions. Dover: New York, 1972.

[26] L. Li, F. Li, F.B. Gross, ”A new polynomial approximation for JV Bessel functions," Applied Mathematics and Computation, pp. 1220 - 1225, Jan. 2005.

[27] I.S. Gradshteyn, I.M. Ryzhik, Table of Integrals, Series, and Products, 6th Ed. Academic Press: London, 2000.

[28] A. Castano-Martinez, F. Lopez-Blazquez, "Distribution of a sum of weighted noncentral chi-square variables," TEST, vol. 14, pp. 397 415,2005

[29] H. Liu, Y. Tang, H.H. Zhang, "A new chi-square approximation to the distribution of non-negative definite quadratic forms in non-central normal variables," Computational Statistics and Data Analysis, vol. 53, pp. $853-856,2009$.

[30] M. Bibinger, "Notes on the sum and maximum of independent exponentially distributed random variables with different scale parameters," http://arxiv.org/abs/1307.3945, 2013.

[31] S. Sanders and A. Aragon-Zavala, Antennas and Propagation for Wireless Communication Systems, 2dn Ed. Wiley: Chichester, UK, 2007.

[32] A. Abdi, M. Kaveh, "K distribution: an appropriate substitute for Rayleigh-lognormal distribution in fading shadowing wireless channels," Electronics Letters, vol. 34, pp. 851 - 852, Apr. 1998.

[33] P.S. Bithas, P.T. Mathiopoulos, S.A. Kotsopoulos, "Diversity reception over generalized-K (KG) fading channels," IEEE Trans. Wireless Commun., vol. 6, pp. 4238 - 4243, Dec. 2007. 
Table I

MAXIMUM AVERAGE HARVESTED POWER IN MW AND OPTIMUM MAGNITUDES.

\begin{tabular}{|c|c|c|c|c|}
\hline model & one harvester two sources & one harvester three sources & two harvesters two sources & three harvesters three sources \\
\hline \hline LEH, fading & $0.14(0.46)$ & $0.25(0.33,0.54)$ & $0.12(0)$ & $0.18(0,0)$ \\
\hline NLEH, fading & $0.16(0.52)$ & $0.22(0.40,0.57)$ & $0.14(0.46)$ & $0.20(0.11,0.64)$ \\
\hline \hline LEH, shadow/fading & $0.14(0.46)$ & $0.25(0.33,0.54)$ & $0.12(0)$ & $0.18(0,0)$ \\
\hline NLEH, shadow/fading & $0.15(0.51)$ & $0.20(0.31,0.60)$ & $0.13(0.47)$ & $0.19(0.09,0.64)$ \\
\hline
\end{tabular}

[34] P.G. Moschopoulos, "The distribution of the sum of independent Gamma random variables," Ann. Inst. Statist. Math., vol. 37, pp. 541 - 544, 1985.

[35] G.H. Golub, "Some modified eigenvalue problems," SIAM Review, vol. 15, pp. 318 - 334, 1973.

[36] X. Zhou, R. Zhang, C.K. Ho, "Wireless information and power transfer: architecture design and rate-energy tradeoff," IEEE Trans. Commun., vol. 61, pp. 4754 - 4767, November 2013.

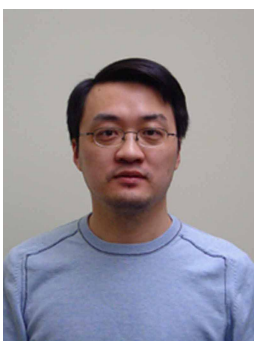

Yunfei Chen (S'02-M'06-SM'10) received his B.E. and M.E. degrees in electronics engineering from Shanghai Jiaotong University, Shanghai, P.R.China, in 1998 and 2001, respectively. He received his $\mathrm{Ph}$.D. degree from the University of Alberta in 2006. $\mathrm{He}$ is currently working as an Associate Professor at the University of Warwick, U.K. His research interests include wireless communications, cognitive radios, wireless relaying and energy harvesting.

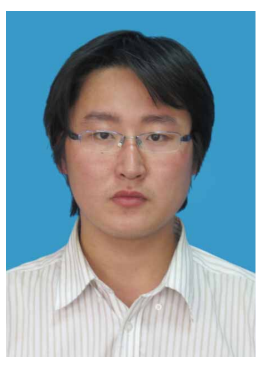

Nan Zhao (S'08-M'11-SM'16) is currently an Associate Professor in the School of Information and Communication Engineering at Dalian University of Technology, China. He received the B.S. degree in electronics and information engineering in 2005, the M.E. degree in signal and information processing in 2007, and the Ph.D. degree in information and communication engineering in 2011, from Harbin Institute of Technology, Harbin, China. His recent research interests include interference alignment, wireless power transfer, and physical layer security.

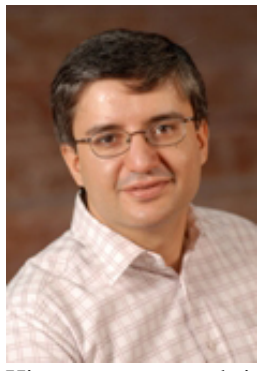

Mohamed-Slim Alouini (S'94-M'98-SM'03-F'09) was born in Tunis, Tunisia. He received the Ph.D. degree in Electrical Engineering from the California Institute of Technology (Caltech), Pasadena, CA, USA, in 1998. He served as a faculty member in the University of Minnesota, Minneapolis, MN, USA, then in the Texas A\&M University at Qatar, Education City, Doha, Qatar before joining King Abdullah University of Science and Technology (KAUST), Thuwal, Makkah Province, Saudi Arabia as a Professor of Electrical Engineering in 2009. His current research interests include the modeling, design, and performance analysis of wireless communication systems. 